\title{
Azithromycin Treatment for SARS-CoV-2-related COVID-19 Pandemic Could Worsen Extensively Drug Resistant (XDR) Typhoid: A Risk of Losing the Last Bullet Against Salmonella enterica Serovar Typhi
}

\author{
Umar Saeed ${ }^{1,{ }^{*}}$, Sara Rizwan Uppal ${ }^{1}$, Zahra Zahid Piracha ${ }^{1}$ and Rizwan Uppal ${ }^{1}$ \\ ${ }^{1}$ Islamabad Diagnostic Center, Islamabad, Pakistan \\ "Corresponding author: Islamabad Diagnostic Center, Islamabad, Pakistan. Email: umarsaeed15@yahoo.com \\ Received 2021 February 17; Revised 2021 April 14; Accepted 2021 April 18.
}

Keywords: COVID-19, SARS-CoV-2, Extensively Drug Resistant (XDR) Typhoid, Salmonella enterica Serovar Typhi

\section{Dear editor,}

Viral infections are increasing day by day (1-3). Severe acute respiratory syndrome (SARS) coronavirus 2 (SARS$\mathrm{CoV}-2$ ) pandemic has resulted in the negligence of diseases such as diabetes, heart diseases, and many more. Among these neglected diseases is extensively drug resistant (XDR) typhoid fever. Intensive care units in hospitals are hotspots for antimicrobial resistance (4). Although antibiotics do not inhibit SARS-CoV-2 replication, yet viral respiratory infections may lead to bacterial pneumonia (4). Hydroxychloroquine and azithromycin have been frequently used for COVID-19 ailment based upon small uncontrolled studies, which showed preliminary effective results. Azithromycin is commonly used to potentially treat or prevent co-infection with SARS-CoV-2. It has been reported that azithromycin may have antiviral properties against RNA viruses such as Zika, rhinovirus, and SARS-CoV$2(4-7)$.

In Pakistan, since 2016, there have been several outbreaks of antimicrobial resistant strains of Salmonella enterica serovar Typhi belonging to the H58 haplotype that cause XDR typhoid fever (8). The H58 strains are resistant to chloramphenicol, ampicillin, and trimethoprimsulfamethoxazole antibiotics (9). Salmonella enterica serovar Typhi has shown resistance to fluoroquinolones and ceftriaxone (10), while orally only azithromycin antibiotic has shown significant responses for the treatment of XDR typhoid. In Pakistan, azithromycin has been given to treat COVID-19 patients while neglecting the prevalence of XDR typhoid fever. This might bring havoc in the near future if XDR becomes resistant against azithromycin. Of note, XDR typhoid fever has been extensively misdiagnosed before due to serological tests such as Widal and
Typhi DOT, which are still in practice by several physicians, despite the fact that the National Institute of Health has clearly directed to stop the diagnosis of typhoid fever via serological tests (11).

Contemplating the importance of phages and lytic enzyme biology might facilitate designing novel therapeutic options against multiple drug-resistant bacteria (12). Azithromycin is neither the cure for COVID-19 nor can it block SARS-CoV-2 replication. In several countries, it has been empirically prescribed by many physicians to save lives. However, the unnecessary usage of azithromycin should be immediately discontinued because it is the only oral antibiotic left that is effective against XDR typhoid. Development of azithromycin resistance in XDR typhoid may result in future epidemics of potentially untreatable typhoid. The need of the hour is also to explore further therapeutic options such as phage therapy against XDR typhoid fever.

\section{Footnotes}

Authors' Contribution: RU and US conceived the study. $\mathrm{RU}$ is the principal investigator (PI) of the study. US wrote the manuscript and analyzed the data and Co-PI of this study. SRU and ZZP assisted in manuscript writing and data analysis.

Conflict of Interests: The authors have no conflicts of interest.

Ethical Approval: The manuscript was approved by the ethical board of IDC for publication.

Funding/Support: No funding was allotted for this study. 


\section{References}

1. Saeed U, Uppal SR, Piracha ZZ, Rasheed A, Aftab Z, Zaheer H, et al. Evaluation of SARS-CoV-2 antigen-based rapid diagnostic kits in Pakistan: Formulation of COVID-19 national testing strategy. Virol J. 2021;18(1):34. doi: 10.1186/s12985-021-01505-3. [PubMed: 33581714]. [PubMed Central: PMC7881305].

2. Saeed U, Uppal SR, Piracha ZZ, Uppal R. COVID-19 transmission via fomites at low temperature: A potential silent SARS-CoV-2 propagation route. Am J Biomed Sci \& Res. 2021;12(1):80-2.AJBSR.MS.ID.001716. doi: 10.34297|AJBSR.2021.12.001716.

3. Saeed U, Uppal SR, Piracha ZZ, Khan AA, Rasheed A, Zaheer H, et al. Effectivity analysis of SARS-CoV-2 nasopharyngeal swab rapid testing kits in Pakistan: A scenario of inadequate COVID-19 diagnosis. Research Square. 2021. doi: 10.21203/rs.3.rs-315851/v1.

4. Reardon S. Antibiotic treatment for COVID-19 complications could fuel resistant bacteria. Science. 2020. doi: 10.1126/science.abc2995.

5. Retallack H, Di Lullo E, Arias C, Knopp KA, Laurie MT, SandovalEspinosa $\mathrm{C}$, et al. Zika virus cell tropism in the developing human brain and inhibition by azithromycin. Proc Natl Acad Sci $U$ S A. 2016;113(50):14408-13. doi: 10.1073/pnas.1618029113. [PubMed: 27911847]. [PubMed Central: PMC5167169].

6. Schogler A, Kopf BS, Edwards MR, Johnston SL, Casaulta C, Kieninger E, et al. Novel antiviral properties of azithromycin in cystic fi- brosis airway epithelial cells. Eur Respir J. 2015;45(2):428-39. doi: 10.1183/09031936.00102014. [PubMed: 25359346].

7. Oldenburg CE, Doan T. Azithromycin for severe COVID-19. Lancet. 2020;396(10256):936-7. doi: 10.1016/S0140-6736(20)31863-8. [PubMed: 32896293]. [PubMed Central: PMC7833672].

8. World Health Organization. Typhoid fever - Islamic Republic of Pakistan. Geneva, Switzerland: World Health Organization; 2018, [cited 2021]. Available from: https://www.who.int/csr/don/27-december2018-typhoid-pakistan/en/.

9. Dyson ZA, Klemm EJ, Palmer S, Dougan G. Antibiotic resistance and typhoid. Clin Infect Dis. 2019;68(Suppl 2):S165-70. doi:10.1093/cid/ciy1111. [PubMed: 30845331]. [PubMed Central: PMC6405283].

10. Qureshi S, Naveed AB, Yousafzai MT, Ahmad K, Ansari S, Lohana H, et al. Response of extensively drug resistant Salmonella Typhi to treatment with meropenem and azithromycin, in Pakistan. PLoS Negl Trop Dis. 2020;14(10). e0008682. doi: 10.1371/journal.pntd.0008682. [PubMed: 33057330]. [PubMed Central: PMC7561124].

11. National Institute of Health. Field epidemiology $\&$ disease surveillance division. Islamabad, Pakistan: National Institute of Health; 2020, [cited 2021]. Available from: https://www.nih.org.pk/wpcontent/uploads/2020/07/Advisory-for-Prevention-andTreatment-of-Typhoid-Fever-including-XDR-Typhoid.pdf.

12. Piracha Z, Saeed U, Khurshid A, Chaudhary WN. Isolation and partial characterization of virulent phage specific against Pseudomonas aeruginosa. Glob J Med Res. 2014;14(1):1-8. 